\title{
Fabrication of potential gastroretentive microspheres of itraconazole for stomach-specific delivery: Statistical optimization and in vitro evaluation
}

\author{
Surbhi Rohilla*, Dinesh Chander Bhatt, Shaveta Ahalwat \\ Department of Pharmaceutical Sciences, Guru Jambheshwar University of Science and Technology, Hisar, Haryana 125001, India.
}

\begin{tabular}{l}
\hline ARTICLE INFO \\
\hline Received on: $26 / 04 / 2019$ \\
Accepted on: $27 / 01 / 2020$ \\
Available online: $05 / 03 / 2020$
\end{tabular}

\section{Key words:}

Hollow microspheres,

Eudragit RS100, ethyl

cellulose, optimization,

central composite design.

\begin{abstract}
The premise of the study was to develop and optimize multiple unit gastroretentive microspheres of itraconazole to prolong its localization in the stomach and analyzed using response surface methodology. The emulsion solvent diffusion evaporation method was used to prepare hollow microsphere of ethyl cellulose and Eudragit RS100 as lowdensity shell-forming polymers. The experimental design matrix was prepared using a central composite design to study the effect of various process parameters over response variables. The optimized microspheres showed a particle size of $285.1 \mu \mathrm{m}$, drug entrapment efficiency of $86.8 \%$, buoyancy of $51.1 \%$, and cumulative drug release of $77.80 \%$. The experimental responses were in good harmony with the predicted values. The compatibility between drug and excipients was determined by Fourier-transform infrared and differential scanning calorimetry analysis. The results signify that gastroretentive hollow microspheres are a promising vehicle to extend the retention time of itraconazole in the upper GI tract, and it can be floated in an acidic medium for a prolonged period.
\end{abstract}

\section{INTRODUCTION}

Candida spp. are harmless commensals or normal occupants of the mucosal surface and become opportunistic pathogens in those individuals who are immunologically weak and immunocompromised (Rohilla et al., 2018). Since the 1980s, the incidence of invasive fungal infection due to Candida spp. increases by nearly 500\% (Gajra et al., 2015).

Itraconazole comes under triazole category of an antifungal agent having broad-spectrum activity used for the treatment of superficial and systemic fungal infections. Many fungal infections require systemic antifungal therapy for their successful treatment (Boogaerts et al., 2001; De Beule and Van Gestel, 2001).

*Corresponding Author

Surbhi Rohilla, Department of Pharmaceutical Sciences, Guru Jambheshwar University of Science and Technology,

Hisar, Haryana 125001, India.

E-mail: surbhiraman.rohilla22@gmail.com
As per the Biopharmaceutics Classification System, itraconazole is having low solubility and high permeability, i.e., it belongs to class II, a highly lipophilic molecule. It is a weakly basic drug $(\mathrm{pKa}$ value $=3.7)$ having $\mathrm{pH}$-dependent dissolution (Abuhelwa et al., 2015). Thus, it has a narrow absorption window and has a need of an acidic environment for adequate dissolution and sufficient absorption of drug. These characteristics make oral absorption of itraconazole difficult (Dominguez-Gil Hurle et al., 2006).

The oral route is most convenient, well-known, and commonly used route for drug delivery. The development of stomach-specific oral drug delivery is a challenging work as rapid gastrointestinal transit can decrease the drug absorption at its absorption site which reduces the efficiency of the administered dose (Tadros, 2010). A site-specific drug delivery is a recent focus of research efforts for drug release in a certain region. Therefore, gastric retention time can be lengthen by gastroretentive drug delivery systems which retain it in the gastric region for a prolonged period, to improve the oral bioavailability of the basic drugs (Farooq et al., 2017; Nila et al., 2014). Above all the various gastroretentive techniques, floating drug delivery system 
is the most important to maintain the drug in the gastric region for a longer period. Hollow microspheres, a type of floating drug delivery systems, with properties such as high specific surface area, low density, and good flowability, have attracted attention toward it (Wang et al., 2007).

The response surface methodology is renowned approach in support of experimental designing and optimization of pharmaceutical formulations with minimum experimentation. Based on the design of experiments, it generates significant polynomial equations to validate the statistical design (Mishra et al., 2016; Shah and Pathak, 2010). The polynomial models can be used to envisage the correlation between input data and response variables by summarizing experiential data (Chaibva and Walker, 2012). Central composite design (CCD) is one of the techniques in response surface methodology, which allows the minimum number of experimental runs and picks the optimal composition for accomplishing the desired goal (Hao et al., 2012).

In this study, gastroretentive hollow microspheres were formulated for itraconazole, a poorly water-soluble drug having a narrow absorption window by employing emulsion solvent diffusion evaporation method for prolonged buoyancy to retain the delivery system at its absorption site to increase its rate and extent of absorption. The formulation variables were optimized by employing CCD. The selected independent variables, such as ratio of polymers and concentration of polyvinyl alcohol, were measured for their resultant effect on responses such as particle size, entrapment efficiency, buoyancy, and \% cumulative drug release. The hollow microspheres were characterized by X-ray diffraction study, differential scanning calorimetry (DSC), micrometric properties, and scanning electron microscopy (SEM).

\section{MATERIALS AND METHODS}

\section{Materials}

Itraconazole was procured as a gift sample from the Zydus Cadila Healthcare Ltd., Ahmedabad, India. Eudragit RS100 was obtained as a gift sample from Evonik Degussa India Pvt. Ltd., Mumbai, India. Ethyl cellulose, dichloromethane (DCM), tween 80, and polyvinyl alcohol were obtained from Thomas Baker Pvt. Ltd., New Delhi. All the remaining reagents and solvents used were of analytical grade.

\section{Methods}

\section{Preparation of microspheres}

The emulsion solvent diffusion evaporation method was used to prepare hollow microspheres (Kawashima et al., 1992). The polymer composed of ethyl cellulose (EC) and Eudragit RS100. Briefly, drug and polymer were dissolved simultaneously in ethanol and DCM mixture (1:1). Then, the dispersion was added dropby-drop into $30 \mathrm{ml}$ polyvinyl alcohol (PVA) solution containing tween $80(0.2 \% \mathrm{w} / \mathrm{v})$, forming oil-in-water $(\mathrm{o} / \mathrm{w})$ emulsion, and stirred on propeller-type mechanical stirrer for 2 hours at $350 \mathrm{rpm}$ and at a temperature of $40^{\circ} \mathrm{C}$ maintained throughout the process. The hollow cavity was formed by evaporation of DCM inside the polymer droplet. The hollow microspheres were separated by filtration and then washed thrice with distilled water, followed by drying at room temperature for 24 hours and storing in a desiccator (Kawashima et al., 1991; Sato et al., 2003; 2004).

\section{Experimental design}

Response surface methodology is a tool to scrutinize the effect of a wide range of independent variables on response variables with the collection of statistical and mathematical techniques. The CCD was adopted to optimize the responses and to find out optimum process parameters. The ratio of polymers $\left(X_{1}\right)$ and concentration of PVA $\left(X_{2}\right)$ were considered as independent variables in the preparation of hollow microspheres. Each factor was studied at three levels $(-1,0$, and +1$)$ as given in Table 1 . The CCD suggested 13 runs, composed of eight factorial points plus five center points to determine the optimum concentrations of independent variables.

The response variables selected were particle size $\left(Y_{1}\right)$, drug entrapment efficiency $\left(Y_{2}\right)$, percentage buoyancy $\left(Y_{3}\right)$, and percentage cumulative drug release $\left(Y_{4}\right)$. Design-Expert software ${ }^{\circledR}$ (version 11.0.0.5, Stat-Ease, Inc., Minneapolis, MN) was used to perform the statistical analysis. The total runs suggested by the design matrix were carried out randomly to eliminate the effects of extraneous or nuisance variables. The design of the experimental matrix and investigated responses are presented in Table 2.

\section{CHARACTERIZATION OF HOLLOW MICROSPHERES}

\section{Particle size analysis}

The particle size analyzer (Microtrac S3500, USA) equipped with a liquid handling system was used to determine the particle size distribution of the microspheres.

\section{Morphology}

The shape and surface morphology of hollow microsphere were determined using scanning electron microscope (EVO18 Zeiss, USA). The samples were prepared by sprinkling a small amount of formulation on a double-adhesive tape stuck to an aluminum stub for gold coating using a gold sputter module to a thickness of $\sim 300 \mathrm{~A}^{\circ}$ under an argon atmosphere in a highvacuum evaporator. The gold-coated samples were then scanned, and micrographs were taken randomly.

\section{Percentage yield}

The percentage yield was calculated by taking the actual weight of dried hollow microspheres by total weight of all nonvolatile excipients used in the formulation of hollow

Table 1. Levels of process variables.

\begin{tabular}{llccccc}
\hline \multirow{2}{*}{ Code } & Process variable & & \multicolumn{2}{c}{ Levels } \\
\cline { 3 - 6 } & & $-\boldsymbol{\alpha}$ & $\mathbf{- 1}$ & $\mathbf{0}$ & $+\mathbf{\alpha}$ \\
\hline$X_{1}$ & Ratio of polymers (EC: Eudragit RS100) & $1.68: 1$ & $2: 1$ & $4: 1$ & $6: 1$ \\
$X_{2}$ & Concentration of PVA (\%w/v) & 0.4 & 0.5 & 0.75 & 1.0 \\
\hline
\end{tabular}


Table 2. Central composite design with the effect of process variables on response values.

\begin{tabular}{|c|c|c|c|c|c|c|}
\hline Code & Polymer Ratio & $\begin{array}{c}\text { Concentration of PVA } \\
(\% \mathrm{w} / \mathrm{v})\end{array}$ & $\begin{array}{l}\text { Particle Size } \\
(\mu \mathrm{m})\end{array}$ & $\begin{array}{c}\text { Entrapment } \\
\text { efficiency }(\%)\end{array}$ & Buoyancy (\%) & $\begin{array}{c}\text { Cumulative drug } \\
\text { release }(\%)\end{array}$ \\
\hline$F_{1}$ & 0 & 0 & 305.5 & 90.77 & 56.72 & 70.15 \\
\hline$F_{2}$ & -1.41421 & 0 & 231.8 & 80.72 & 58.66 & 82.40 \\
\hline$F_{3}$ & 0 & 0 & 284.2 & 87.82 & 52.6 & 71.06 \\
\hline$F_{4}$ & 0 & 0 & 308.2 & 88.4 & 55.59 & 68.54 \\
\hline$F_{5}$ & 1 & -1 & 325.3 & 98.27 & 72.41 & 67.06 \\
\hline$F_{6}$ & 1 & 1 & 276.5 & 86.26 & 59.17 & 78.36 \\
\hline$F_{7}$ & 0 & 0 & 293.3 & 90.11 & 54.04 & 71.72 \\
\hline$F_{8}$ & -1 & 1 & 202.4 & 79.79 & 38.98 & 79.35 \\
\hline$F_{9}$ & 0 & 1.41421 & 223.8 & 83.49 & 45.75 & 80.63 \\
\hline$F_{10}$ & -1 & -1 & 245.5 & 81.64 & 61.41 & 73.62 \\
\hline$F_{11}$ & 1.41421 & 0 & 383.5 & 96.43 & 75.05 & 70.38 \\
\hline$F_{12}$ & 0 & 0 & 278.7 & 86.41 & 48.22 & 72.54 \\
\hline$F_{13}$ & 0 & -1.41421 & 374.2 & 92.11 & 71.0 & 65.14 \\
\hline
\end{tabular}

microspheres (Patel et al., 2006). The percentage yield equation is given as follows:

$$
\% \text { Yield }=\frac{\text { Weight of dried hollow microspheres }}{\text { Total weight of drug and nonvolatile }} \times 100
$$

\section{Micromeritic properties}

The prepared microspheres were characterized for their micromeritic properties, such as bulk density, tapped density, Hausner's ratio, and Carr's compressibility index. The tapping method was adopted to calculate the tapped density (Agrawal et al., 2017). Graduated measuring cylinder was used to measure the bulk and tapped densities. The initial volume (bulk) was noted after pouring the sample into the cylinder, and after 100 tapings, the final tapped volume was again noted.

The formula of the ratio of weight and their respective volume was used to calculate the bulk density and tapped density. Hausner's ratio and Carr's compressibility index of microspheres were computed using the following formula (Garud and Garud, 2012):

Hausner's ratio $=\frac{\text { Tapped density }}{\text { Bulk density }}$

$\begin{aligned} & \text { Carr's compressibility } \\ & \text { index }(\%)\end{aligned}=\frac{\text { Tapped density }- \text { Bulk density }}{\text { Tapped density }} \times 100$

\section{Solubility study}

The saturation solubility of the drug in a given solvent was determined using shake-flask method. The excess amount of drug was placed in simulated gastric fluid (SGF, $50 \mathrm{ml}, \mathrm{pH}$ 1.2). The mixture was placed on a mechanical shaker at room temperature for 24 hours (Maghraby and Alomrani, 2009). The samples were then filtered through a nylon syringe filter $(0.45 \mu \mathrm{m})$ and analyzed using UV spectrophotometer (Cary 5000, Varian, Australia) at $258 \mathrm{~nm}$.

\section{Percentage of drug content and entrapment efficiency}

The percentage of drug content and entrapment efficiency was computed by determining the proportion of the drug that got entrapped in microspheres. For this, a known amount of drug containing microspheres was dissolved in methanol by ultrasonication (Gaur et al., 2014; Jagtap et al., 2012; Sato et al., 2004). The samples were filtered through a $0.45-\mu \mathrm{m}$ nylon filter to remove shell debris. After appropriate dilution, the samples were assayed for drug content and entrapment efficiency at $262 \mathrm{~nm}$ using UV-visible spectrophotometer (Cary 5000, Varian, Australia).

$\%$ Drug Content $=\frac{\text { Weight of drug in microspheres }}{\text { Weight of microspheres }} \times 100$

$\%$ Entrapment Efficiency $=\frac{\text { Actual drug content }}{\text { Theoretical drug content }} \times 100$

\section{In vitro Buoyancy study}

The buoyancy of hollow microspheres was determined using United States Pharmacopoeia (USP) paddle-type dissolution apparatus agitated by a paddle at $100 \mathrm{rpm}$. The amount of microspheres $(100 \mathrm{mg})$ were weighed accurately, and then spread over the surface of $200 \mathrm{ml}$ dispersing medium consist of simulated gastric fluid ( $\mathrm{pH} 1.2$ ) containing $0.5 \% \mathrm{w} / \mathrm{v}$ tween 80 at $37^{\circ} \mathrm{C} \pm 0.5^{\circ} \mathrm{C}$ (Sato et al., 2004; Umamaheswari et al., 2002). After 10 hours, the floating and settled portions were collected separately, dried, and weighed. The buoyancy of the hollow microspheres was represented by the following equation:

$$
\% \text { Buoyancy }=\frac{\text { Weight of hollow microspheres }}{\begin{array}{c}
\text { Total weight of hollow and } \\
\text { settled microsphere }
\end{array}} \times 100
$$

\section{In vitro drug release}

The in vitro drug release from microspheres was carried out using USP paddle-type apparatus. The microspheres equivalent to $30 \mathrm{mg}$ of drug were packed into dialysis membrane and 
immersed in dissolution medium consisting of $900 \mathrm{ml}$ simulated gastric fluid $(\mathrm{pH} 1.2)$ containing $0.5 \% \mathrm{w} / \mathrm{v}$ tween 80 having a paddle rotation speed of $100 \mathrm{rpm}$ at $37^{\circ} \mathrm{C} \pm 0.5^{\circ} \mathrm{C}$ temperature. The samples were withdrawn at suitable time intervals up to 10 hours, filtered through a $0.45 \mu \mathrm{m}$ membrane filters, and analyzed UV spectrophotometrically at $258 \mathrm{~nm}$. Sink conditions prevailed during the dissolution studies. All the experiments were carried out in triplicate.

\section{Kinetics of drug release}

The data obtained from in vitro analysis were fitted to various mathematical models to determine the mechanism of drug release (Costa and Lobo, 2001; Dash et al., 2010) such as zeroorder kinetics (percentage release vs. time), first-order kinetics (log percentage release $v s$. time), Higuchi's model (percentage release $v s$. square root of time), and Korsmeyer-Peppas model (log cumulative percentage drug release vs. log time).

\section{Fourier-transform infrared spectroscopy analysis}

Fourier-transform infrared (FT-IR) analysis was performed to determine the possibility of any chemical interaction between drug and excipients. For this, the samples were reduced to a fine powder, mixed with $\mathrm{KBr}$, and placed it over the powder sample holder. The spectrum scan was done in frequency range of 4,000-400 $\mathrm{cm}^{-1}$ using a FT-IR spectrophotometer (IR Affinity, Shimadzu, Japan).

\section{Differential scanning calorimetric analysis}

The thermal analysis of samples was done using differential scanning calorimeter (DSC 25, TA instruments, CA). Samples of 2.5-5 mg were placed into an aluminum pan and raised the temperature at a constant rate $\left(10^{\circ} \mathrm{C}\right.$ minute $\left.{ }^{-1}\right)$, from $30^{\circ} \mathrm{C}$ to $300^{\circ} \mathrm{C}$ under nitrogen atmosphere.

\section{X-ray diffraction study}

The X-ray diffraction analysis was performed using X-ray diffractometer (Rigaku Miniflex-II X-ray diffractometer, Japan). The powder diffractometer sets for the continuous scan with an angle range of $10^{\circ}-70^{\circ} 2 \theta$ and step size of $0.04^{\circ} 2 \theta$. The analysis was carried out on itraconazole, polymers empty the hollow microspheres, and itraconazole loaded the hollow microspheres.

\section{RESULTS AND DISCUSSION}

\section{Preparation and optimization of hollow microspheres}

The hollow microspheres are multiple unit drug delivery systems and are the most efficient form of delivery systems as they can be extensively distributed throughout the stomach and may provide efficient release of drugs at the target site. Floating hollow microspheres were prepared by emulsion solvent diffusion evaporation method. Using above technique, the polymeric microsphere was prepared with an internal spherical hollow cavity produced by diffusion and subsequent evaporation of solvent mixture. The ethanol present in polymer droplet diffused out into water; thus, polymer-drug mixture was solidified at DCM droplet (Gupta et al., 2014), which got evaporated on stirring at $40^{\circ} \mathrm{C} \pm$ $5^{\circ} \mathrm{C}$ (Sato et al., 2003). The effect of independent variables such as the ratio of polymers and concentration of surfactant was studied on the physicochemical properties of the hollow microspheres such as particle size, entrapment efficiency, buoyancy, and cumulative drug release employing experimental CCD. In response surface methodology, various empirical models are fitted to experimental data which depend on the experimental design. In this study, the CCD was selected as an experimental design, as it adequately describes the interaction between the factors with the least number of experiments. The preliminary studies were conducted to ascertain the levels of independent variables for optimization (Bezerra et al., 2008, Liu et al., 2014).

The Design-Expert software computed the statistical parameters, and it indicated that all responses generated using experimental design were best fitted into various polynomial models. The observed responses, i.e., particle size $\left(Y_{1}\right)$ and entrapment efficiency $\left(Y_{2}\right)$, were fitted best into linear and 2FI response surface model, respectively ( $p$-value 0.0003 for $Y_{1}$ and $p$-value $<0.0001$ for $Y_{2}$ ), whereas percentage buoyancy $\left(Y_{3}\right)$ and percentage cumulative drug release $\left(Y_{4}\right)$ are fitted best into quadratic response surface model ( $p$-value 0.0007 for $Y_{3}$ and $p$-value 0.0006 for $Y_{4}$ ) with no transformations of data. The polynomial equations for all dependent variables are as follows:

$$
\begin{aligned}
Y_{1}(\mu \mathrm{m})= & 287.15+46.05 X_{1}-38.07 X_{2} \\
Y_{2}(\%)= & 87.8631+5.66466 X_{1}-3.25632 X_{2}-2.54 X_{1} X_{2} \\
Y_{3}(\%)= & 53.434+6.79612 X_{1}+-8.92236 X_{2}+2.2975 X_{1} X_{2}+ \\
& 5.55487 X_{1}^{2}+1.31488 X_{2}^{2} \\
Y_{4}(\%)= & 70.802-3.06861 X_{1}+4.86702 X_{2}+1.3925 X_{1} X_{2}+ \\
& 2.784 X_{1}^{2}+1.0315 X_{2}^{2}
\end{aligned}
$$

The above polynomial equations indicated the correlation between process variables and responses. Table 3 represents the effect of factors on responses and their associated $p$-values. Any model was considered to be significant if the $p$-value $<0.05$ at $95 \%$ confidence level. The positive and negative signs depict the synergistic effect and antagonistic effect, respectively, which is having a relative impact of each factor on the responses.

Table 4 shows the result of analysis of variance analysis on models, and the summary statistics indicated that the response surface model developed for all response variables is significant, having $p<0.05$ with non-significant "lack of fit" ( $p>0.05)$, which ensures the reliability of applied model. The reliability of models was denoted by higher $R_{2}$ values and good agreement between predicted $R_{2}$ and adjusted $R_{2}$. In addition to the above, adequate precision measures the signal-to-noise ratio, and the higher values of adequate precision $(>4)$ signify that models are fitted to navigate the design space.

A three-dimensional response surface model analysis graphs were generated for the optimization of hollow microspheres by response surface methodology. The three-dimensional response surface plots for all the responses are presented in Figure 1. The response surface plot of particle size illustrates the interaction and effect of investigated variables as shown in Figure 1(a). The polymer ratio and concentration of PVA had a pronounced effect on particle size. The particle size decreases with the increase in the concentration of PVA, which reduces the interfacial tension more efficiently, and polymers become more uniformly dispersed in aqueous phase by 
Table 3. Summary of factor effect and associated $p$-values for all responses.

\begin{tabular}{ccccccccc}
\hline \multirow{2}{*}{ Factors } & \multicolumn{2}{c}{$\boldsymbol{Y}_{\mathbf{1}}$} & \multicolumn{2}{c}{$\boldsymbol{Y}_{2}$} & & $\boldsymbol{Y}_{3}$ & & $\boldsymbol{Y}_{4}$ \\
\cline { 2 - 9 } & $\mathbf{E f f e c t s}$ & $\boldsymbol{p}$-values & Effects & $\boldsymbol{p}$-values & Effects & $\boldsymbol{p}$-values & Effects & $\boldsymbol{p}$-values \\
\hline$X_{1}$ & +46.05 & 0.0005 & +5.66 & $<0.0001$ & +6.79 & 0.0012 & -3.068 & 0.0021 \\
$X_{2}$ & -38.07 & 0.0020 & -3.25 & 0.0004 & -8.92 & 0.0002 & +4.86 & 0.0001 \\
$X_{1} X_{2}$ & & & -2.54 & 0.0137 & +2.29 & 0.2512 & +1.39 & 0.1732 \\
$X_{1}{ }^{2}$ & & & & & +5.55 & 0.0053 & +2.78 & 0.0052 \\
$X_{2}{ }^{2}$ & & & & & +1.31 & 0.3765 & +1.03 & 0.1820 \\
\hline
\end{tabular}

Table 4. Detail Summary of statistical analysis of response surface models.

\begin{tabular}{ccccccccccc}
\hline & \multicolumn{1}{c}{ Model } & \multicolumn{4}{c}{ Lack of fit } \\
\hline $\begin{array}{c}\text { Response } \\
\text { factor }\end{array}$ & $\boldsymbol{F}$-value & $\boldsymbol{p}$-value & $\boldsymbol{R}^{\mathbf{2}}$ & Adjusted $\boldsymbol{R}^{\mathbf{2}}$ & Predicted $\boldsymbol{R}^{\mathbf{2}}$ & Adeq. Prec. & $\boldsymbol{C . v .}$ & Std. dev. & $\boldsymbol{F}$-value & $\boldsymbol{p}$-value \\
\hline$Y_{1}$ & 21.14 & 0.0003 & 0.8087 & 0.7705 & 0.6041 & 13.47 & 9.05 & 25.99 & 6.11 & 0.0507 \\
$Y_{2}$ & 44.24 & $<0.0001$ & 0.9365 & 0.9153 & 0.8348 & 21.00 & 1.89 & 1.66 & 0.808 & 0.5977 \\
$Y_{3}$ & 18.45 & 0.0007 & 0.9295 & 0.8791 & 0.6795 & 12.76 & 6.37 & 3.67 & 1.55 & 0.3327 \\
$Y_{4}$ & 19.55 & 0.0006 & 0.9332 & 0.8854 & 0.6736 & 12.72 & 2.51 & 1.84 & 1.99 & 0.2578 \\
\hline
\end{tabular}

stabilization of polymer droplets. The polymer ratio had a positive effect on particle size. The EC has a marked effect compared to Eudragit RS100, which could be related to the viscosity of polymer resulting in high interfacial tension and increased particle size, whereas Eudragit RS100 formed the compact structure as compared to EC (Fartyal et al., 2011; Pandav et al., 2014).

Figure 1(b) represents the response surface curve of entrapment efficiency. The entrapment was directly proportional to polymer concentration and inversely proportional to the concentration of PVA. The microspheres prepared with a higher ratio of EC showed high drug entrapment compared to Eudragit RS100. This may be due to hydrophobic nature of EC, which encapsulates a larger amount of drug as compared to Eudragit RS100 and increases the matrix-forming potential of polymer with drug and decreases the drug diffusion to the external phase. Due to this, the maximum amount of drug was forced to reside in the microspheres formed, resulting in high percentage entrapment (Nour et al., 2015). However, the entrapment efficiency was reduced with an increase in the concentration of PVA. This is possibly due to smaller size microspheres formed at higher concentration.

The effect of process variables on buoyancy can be measured as shown in Figure 1(c). The in vitro buoyancy of microspheres can be correlated to low density and insolubility of polymers in the simulated gastric fluid $(\mathrm{pH} 1.2)$. As reported by Sato et al., the buoyancy of particles depends on their density and size. The size of microspheres exhibited an inverse relationship to the microsphere density. Hence, the buoyancy of microspheres increased with an increase in particle size, which can be directly related to the increase in the ratio of polymers and inversely related to the increase in the concentration of PVA. The microspheres prepared with a high concentration of PVA were smaller in size and less dense than those prepared with a lower concentration of PVA. The density of EC is $0.4 \mathrm{~g} / \mathrm{cc}$, which is lower than Eudragit RS100 (Rowe et al., 2006), so the particles having a higher ratio of EC are less dense and more buoyant compared to Eudragit RS100.

Figure 1(d) illustrates the combined effect of independent variables on the cumulative release of itraconazole. The response surface curve inferred that on increasing the relative fraction of Eudragit RS100, the percentage cumulative drug release increases to some extent as compared to the increase in the proportion of EC. In general, an increase in polymer concentration increased the diffusion path of drug which decreases the overall drug release from the polymer matrix. In contrast with the above statement, larger droplets facilitate a rapid penetration of dissolution medium which indirectly indicates more interconnected pores that help in drug dissolution and facilitate the drug diffusion from the polymeric matrix (Raut et al., 2013; Raghuvanshi et al., 2016). The extent of drug release was predominantly dependent on PVA levels as it increases the wettability of particles and better solvent penetration (Pachuau and Mazumder, 2009). Typically, the formulation made with higher PVA and Eudragit RS100 levels and lower EC level exhibited a high percentage cumulative drug release. Hollow microspheres with desired parameters were accomplished by desirability approach using numerical optimization tool of DesignExpert software. The goal was to obtain the optimum values of the independent variables. Optimization was performed by setting constraints such as maximum particle size, buoyancy, cumulative drug release, and in range entrapment efficiency. The Design-Expert software provided four solutions; the solution with the highest desirability was selected for optimized formulation. The optimized values of parameters obtained were the ratio of polymers $\left(X_{1}\right)$ of $6: 1$ and the concentration of PVA $\left(X_{2}\right)$ of $1.0 \% \mathrm{w} / \mathrm{v}$. The results for the final optimized concentration are tabulated in Table 5.

\section{Characterization of hollow microspheres}

The data of particle size analysis show that the average size of dried microspheres ranged from 202.4 to $383.5 \mu \mathrm{m}$ (Table 2). Depending on the concentration of PVA and ratio of polymers, the formulations $F_{8}$ and $F_{11}$ showed the smallest and largest size of microspheres, respectively. SEM analysis was performed to study the surface morphological and shape of microspheres. Figure 2 shows the scanning electron microscope images of hollow microspheres. It could be concluded from the figure that the microspheres had a smooth spherical surface having 


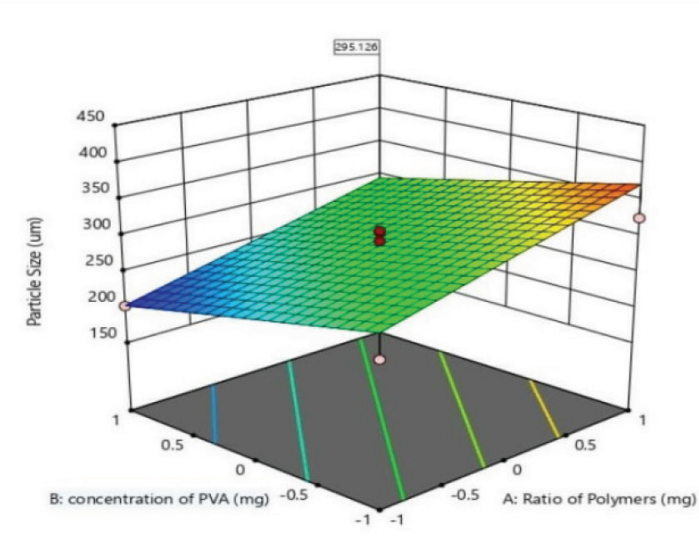

(a)

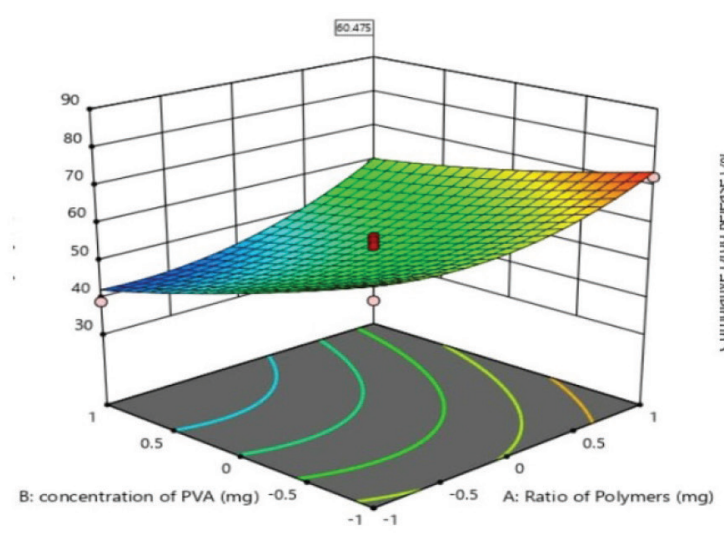

(c)

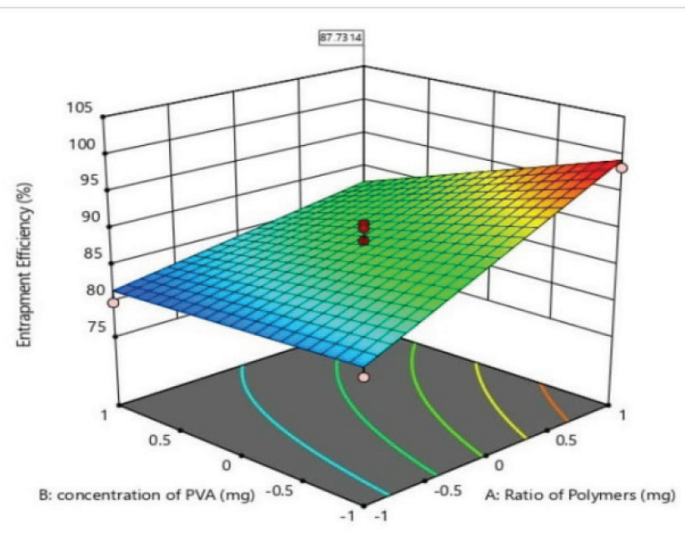

(b)

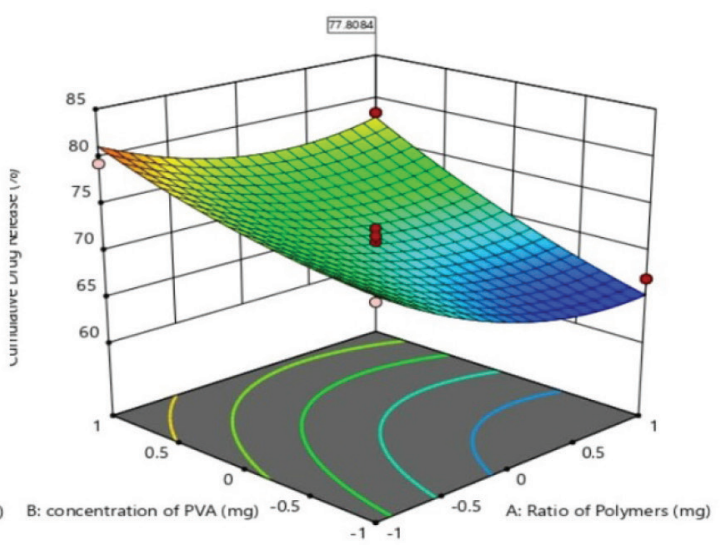

(d)

Figure 1. Response Surface Curve for (a) Particle Size, (b) Entrapment Efficiency, (c) Buoyancy, and (d) Cumulative Drug Release.

Table 5. Observed, predicted values, and percentage prediction error for the responses at optimized concentration.

\begin{tabular}{|c|c|c|c|c|c|c|c|c|c|c|c|c|}
\hline \multirow{2}{*}{$X_{1} / X_{2}$} & \multicolumn{3}{|c|}{$Y_{1}$} & \multicolumn{3}{|c|}{$Y_{2}$} & \multicolumn{3}{|c|}{$Y_{3}$} & \multicolumn{3}{|c|}{$Y_{4}$} \\
\hline & Observed & Predicted & \%Error & Observed & Predicted & \%Error & Observed & Predicted & \%Error & Observed & Predicted & \%Error \\
\hline $6: 1 / 1.0$ & 285.1 & 295.126 & -3.39 & 86.8 & 87.731 & -1.06 & 61.1 & 60.475 & 1.2 & 78.66 & 77.808 & 1.095 \\
\hline
\end{tabular}

pores on them through which dissolution medium could permeate into the drug-polymer matrix and facilitate the diffusion of the drug molecule.

The percentage yield for microspheres was found to be in the range of $46.38 \%-87.33 \%$ as given in Table 6. During microsphere formation, the product yield was affected by the polymers sticking to the wall of the beaker and blades of the stirrer and agglomerate formation. The percentage yield was found to be independent on the ratio of polymers. It decreased with an increase in the concentration of PVA; the reason may be loss of small particles during filtration and washing (Jagtap et al., 2012). The flow properties of the microspheres were studied as Carr's compressibility index and Hausner's ratio. The Carr's compressibility index was in the range of $09.09 \%-20.05 \%$, whereas the Hausner's ratio for all formulations was in the range of 1.04-1.25, which indicated good-to-excellent flow characteristics
(Sinko, 2006). This would help in the handling of microspheres during processing.

The equilibrium solubility of itraconazole was carried out in distilled water and SGF $(\mathrm{pH} 1.2)$ at room temperature. It was recorded to be $\sim 1 \mathrm{ng} / \mathrm{ml}$ in distilled water and $3.35 \pm 0.03$ $\mu \mathrm{g} / \mathrm{ml}$ in simulated gastric fluid $(\mathrm{pH} 1.2)$. The solubility was significantly increased in acidic solution. As shown in Table 6, the drug content increased with the increase in the concentration of emulsifier. The highest drug content was obtained at high polymer ratio and low concentration of PVA. The in vitro release profile of optimized batch is shown in Figure 3. It can be observed from the plot that $78.66 \%$ of the drug was released in a dissolution medium consisting of SGF $\mathrm{pH}$ (1.2) within 10 hours. To determine the mechanism of release kinetics from hollow microspheres, the release rate data were subjected to various kinetic models for the goodness of fit. 


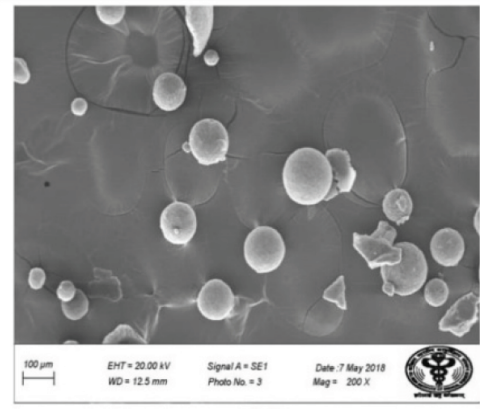

(a)

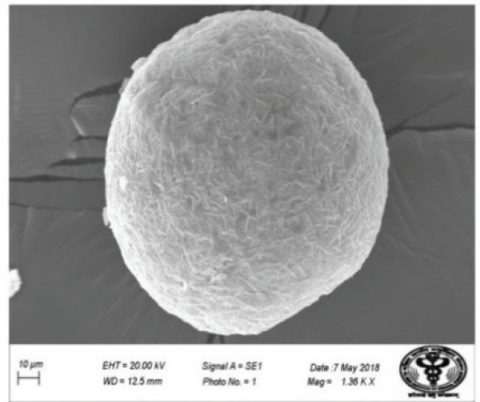

(b)

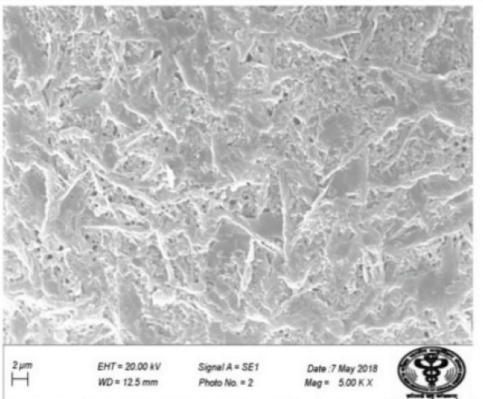

(c)

Figure 2. SEM images of hollow microspheres showing (a) general appearance; (b) single spherical microsphere; and (c) surface morphology.

Table 6. Yield, drug content, and Micromeritic properties (mean \pm SD).

\begin{tabular}{|c|c|c|c|c|c|c|}
\hline Formulation code & Yield (\%) & Drug content $(\%)$ & Bulk density $\left(\mathrm{g} / \mathrm{cm}^{3}\right)$ & Tapped density $\left(\mathrm{g} / \mathrm{cm}^{3}\right)$ & Hausner's ratio & Carr's compressibility index $(\%)$ \\
\hline$F_{1}$ & $66.66 \pm 0.62$ & $14.95 \pm 0.91$ & $0.238 \pm 0.008$ & $0.286 \pm 0.010$ & $1.20 \pm 0.002$ & $16.66 \pm 0.30$ \\
\hline$F_{2}$ & $70.62 \pm 0.76$ & $13.39 \pm 0.44$ & $0.366 \pm 0.012$ & $0.419 \pm 0.014$ & $1.14 \pm 0.003$ & $12.50 \pm 0.22$ \\
\hline$F_{3}$ & $62.12 \pm 0.72$ & $14.57 \pm 0.48$ & $0.22 \pm 0.016$ & $0.260 \pm 0.018$ & $1.18 \pm 0.002$ & $15.38 \pm 0.17$ \\
\hline$F_{4}$ & $60.6 \pm 0.51$ & $14.67 \pm 0.36$ & $0.24 \pm 0.009$ & $0.288 \pm 0.014$ & $1.2 \pm 0.007$ & $16.66 \pm 0.47$ \\
\hline$F_{5}$ & $84.84 \pm 0.81$ & $16.31 \pm 0.57$ & $0.355 \pm 0.015$ & $0.391 \pm 0.020$ & $1.10 \pm 0.004$ & $15.14 \pm 0.38$ \\
\hline$F_{6}$ & $55.55 \pm 0.43$ & $14.32 \pm 0.39$ & $0.226 \pm 0.015$ & $0.235 \pm 0.016$ & $1.04 \pm 0.002$ & $03.99 \pm 0.167$ \\
\hline$F_{7}$ & $65.51 \pm 0.68$ & $14.34 \pm 0.28$ & $0.258 \pm 0.024$ & $0.315 \pm 0.023$ & $1.22 \pm 0.018$ & $18.09 \pm 1.30$ \\
\hline$F_{8}$ & $62.72 \pm 0.56$ & $13.24 \pm 0.36$ & $0.185 \pm 0.017$ & $0.219 \pm 0.022$ & $1.18 \pm 0.006$ & $15.78 \pm 0.38$ \\
\hline$F_{9}$ & $46.38 \pm 0.56$ & $13.86 \pm 0.47$ & $0.214 \pm 0.021$ & $0.275 \pm 0.035$ & $1.25 \pm 0.014$ & $20.05 \pm 0.824$ \\
\hline$F_{10}$ & $85.13 \pm 0.72$ & $13.55 \pm 0.36$ & $0.196 \pm 0.010$ & $0.234 \pm 0.015$ & $1.20 \pm 0.005$ & $16.68 \pm 1.30$ \\
\hline$F_{11}$ & $87.33 \pm 0.93$ & $16.00 \pm 0.59$ & $0.205 \pm 0.008$ & $0.227 \pm 0.012$ & $1.11 \pm 0.002$ & $09.97 \pm 0.216$ \\
\hline$F_{12}$ & $59.39 \pm 0.52$ & $14.32 \pm 0.34$ & $0.220 \pm 0.010$ & $0.260 \pm 0.017$ & $1.18 \pm 0.004$ & $15.38 \pm 0.365$ \\
\hline$F_{13}$ & $83.47 \pm 0.54$ & $15.29 \pm 0.49$ & $0.134 \pm 0.008$ & $0.147 \pm 0.011$ & $1.1 \pm 0.003$ & $09.09 \pm 0.307$ \\
\hline
\end{tabular}

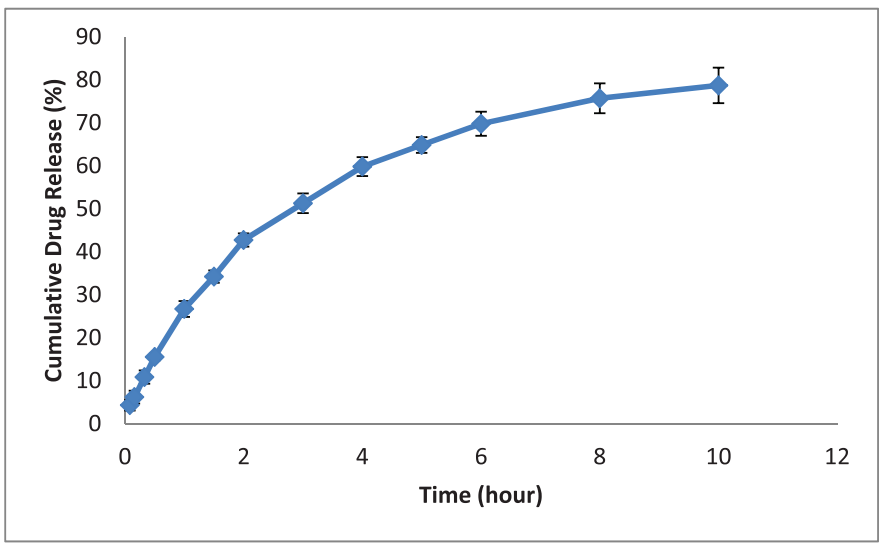

Figure 3. In vitro release profile of optimized batch.

The $R^{2}$ values of release kinetic models were found to be $0.863,0.962,0.985$, and 0.978 for zero-order, first-order, Higuchi's square root, and Korsmeyer-Peppas models, respectively. The results indicated that the in vitro drug release of itraconazole from hollow microspheres was best described by Higuchi's release equation, indicating the release of drug from the insoluble matrix by the diffusion-controlled process.
Figure 4 depicts the FT-IR spectra of itraconazole which showed characteristic peaks occurred at 3,127, 3,068, $2,963,2,877,2,822,1,702,1,654,1,613,1,462,1,451,1,425$, and $418 \mathrm{~cm}^{-1}$. The absorption bands between 2,800 and $3,200 \mathrm{~cm}^{-1}$ represent the stretching of alkane, aromatic rings, and amine groups. The sharp peak occurred at $1,702 \mathrm{~cm}^{-1}$ may be due to $\mathrm{C}=\mathrm{O}$ group, and the peaks at 1,613 and $1,425 \mathrm{~cm}^{-1}$ may be arise due to $\mathrm{C}=\mathrm{N}$ and $\mathrm{C}-\mathrm{N}$ bonds, respectively. All the characteristic peaks of itraconazole were present without any new peak formation in the spectrum of the physical mixture and optimized batch, thus indicating the compatibility between drug and polymers with no chemical interaction.

DSC thermograms of itraconazole, EC, Eudragit RS 100 , physical mixture of drug and excipients, and optimized batch are presented in Figure 5. In the case of itraconazole, a sharp endothermic peak was observed at $167.85^{\circ} \mathrm{C}$, which corresponded to the melting process. Thermograms of ethyl cellulose showed broad endothermic peaks at $56.77^{\circ} \mathrm{C}$ and $184.14^{\circ} \mathrm{C}$ and Eudragit RS100 showed peaks at $65.46^{\circ} \mathrm{C}$ and $184.89^{\circ} \mathrm{C}$, respectively, which correspond to melting process and thermal decomposition of polymers. A physical mixture of drug and excipients showed endothermic peaks at $63.48^{\circ} \mathrm{C}, 165.98^{\circ} \mathrm{C}$, and $186.5^{\circ} \mathrm{C}$; a slight decrease and broadening of the peak of itraconazole by $1.8^{\circ} \mathrm{C}$ were 


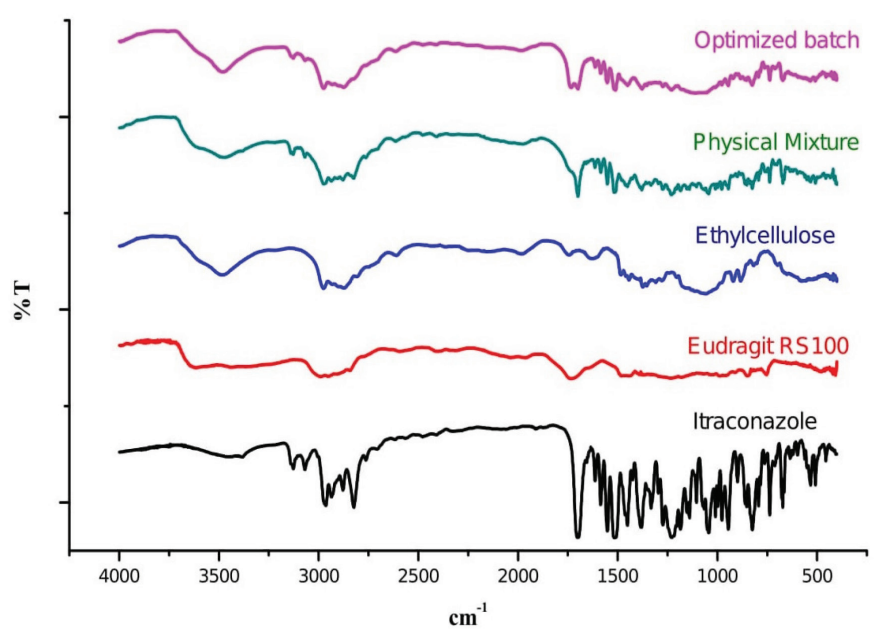

Figure 4. FTIR spectra of Itraconazole; Eudragit RS100; Ethyl cellulose; Physical mixture, and Optimized batch.

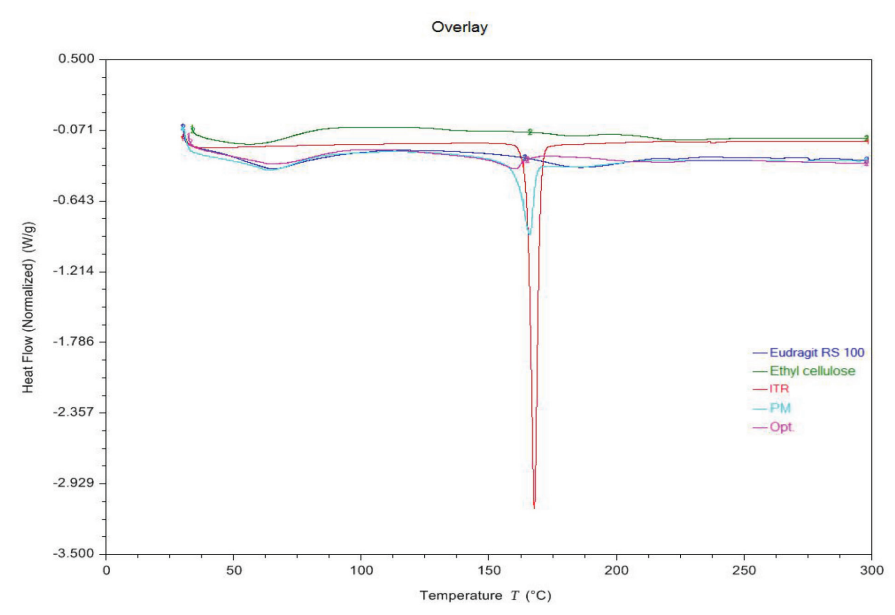

Figure 5. DSC of Eudragit RS 100; Ethyl cellulose; Itraconazole (ITR); Physical mixture (PM), and Optimized batch (Opt).

attributable to the presence of low-melting amorphous excipients, whereas a slight increase in endothermic peaks of polymers was attributed to the high-melting crystalline drug. In the case of DSC curve of optimized batch, a small blunt endothermic peak of itraconazole was observed at $160.31{ }^{\circ} \mathrm{C}$ and further decreased by $5.67^{\circ} \mathrm{C}$ which may be due to reduction in the crystallinity of drug by its solubilization in the polymer matrix. The DSC thermogram of physical mixture signifies that drug had no interaction with polymers used in the formulation as no new peak appeared in it.

The overlay of X-ray diffraction data is listed in Figure 6. The diffractogram of itraconazole showed a peak with a maximum intensity of $10,033.3$ at $2 \theta$ value of $20.46^{\circ}$. The other important peaks are observed at $2 \theta$ values of $14.58^{\circ}, 17.58^{\circ}$, and $23.58^{\circ}$, and their corresponding peak intensities are 4,223, 7,466, and 6,320, respectively. The physical mixture of itraconazole and polymers showed the slight hump of polymers, whereas the peaks of itraconazole were also apparently visible. The diffractogram of drug-loaded hollow microspheres showed a characteristic peak at an angle of $20.34^{\circ}$ with peak intensity 5,160 corresponding to itraconazole. The diffraction pattern of the pure drug showed a

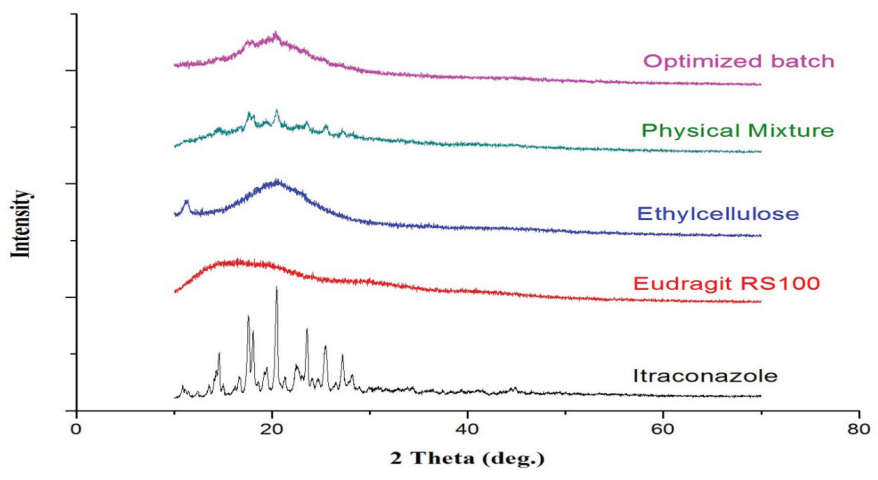

Figure 6. X-ray diffractograms of Itraconazole; Eudragit RS 100; Ethyl cellulose; Physical mixture, and Optimized batch.

remarkable difference from drug-loaded microspheres (optimized batch), and the former showed relative sharp peaks due to high degree of crystallinity of itraconazole. The decrease in peak intensity of the diffraction pattern of itraconazole-loaded hollow microspheres confirmed the change of drug from crystalline to amorphous form.

\section{CONCLUSION}

The results of this study emphasized that response surface methodology with $\mathrm{CCD}$ is a very useful statistical technique to determine the effect of selected independent variables on the dependent variables. Gastroretentive hollow microspheres were prepared successfully using the emulsion solvent diffusion evaporation technique as per design matrix. The SEM results showed that microspheres were spherical having pores on its surface which enhance the floating ability of the particles. The method of preparation was found to be reliable and inexpensive. The results of optimization revealed that the ratio of polymers and concentration of PVA have a significant effect over response variables such as particle size, entrapment efficiency, buoyancy, and percentage cumulative drug release. The low density of microspheres showed well in vitro floating ability and drug release profile. Hence, floating microspheres of itraconazole prolonged the retention time in the stomach, which can ultimately result in improved bioavailability at a much lower dose. The feasibility of the optimization procedure in developing hollow microspheres can be demonstrated by close agreement between the observed responses and predicted values of the optimized formulation.

\section{ACKNOWLEDGMENT}

The authors express gratitude to the Department of Science and Technology, New Delhi, for providing financial assistance to Surbhi Rohilla (IF150924) under "INSPIRE" fellowship and to SAIF, AIIMS, New Delhi, for SEM analysis.

\section{CONFLICT OF INTEREST}

The authors declare that they have no conflicts of interest.

\section{REFERENCES}

Abuhelwa AY, Foster DJ, Mudge S, Hayes D, Upton RN. Population pharmacokinetic modelling of itraconazole and hydroxyl-

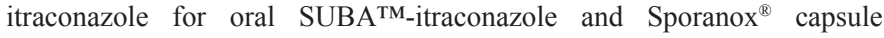
formulations in healthy subjects in fed and fasted states. Antimicrob Agents Chemother, 2015; 59(9):5681-96. 
Agrawal GR, Wakte P, Shelke S. Formulation, physicochemical characterization and in vitro evaluation of human insulin-loaded microspheres as potential oral carrier. Prog Biomater, 2017; 6(3):125-36.

Bezerra MA, Santelli RE, Oliveira EP, Villar LS, Escaleira LA. Response surface methodology (RSM) as a tool for optimization in analytical chemistry. Talanta, 2008; 76(5):965-77.

Boogaerts MA, Maertens J, Van Der Geest R, Bosly A, Michaux JL, Van Hoof A, Cleeren M, Wostenborghs R, De Beule K. Pharmacokinetics and safety of a 7-day administration of intravenous itraconazole followed by a 14-day administration of itraconazole oral solution in patients with hematologic malignancy. Antimicrob Agents Chemother, 2001; 45(3):981-5.

Chaibva FA, Walker RB. The use of response surface methodology for the formulation and optimization of salbutamol sulfate hydrophilic matrix sustained release tablets. Pharm Dev Technol, 2012;17(5):594-606.

Costa P, Lobo JM. Modeling and comparison of dissolution profiles. Eur J Pharm Sci, 2001; 13(2):123-33.

Dash S, Murthy PN, Nath L, Chowdhury P. Kinetic modeling on drug release from controlled drug delivery systems. Acta Pol Pharm, 2010; 67(3):217-3.

De Beule K, Van Gestel J. Pharmacology of itraconazole. Drugs. 2001; 61(1):27-37.

Domínguez-Gil Hurlé A, Sánchez Navarro A, Garcia Sanchez MJ. Therapeutic drug monitoring of itraconazole and the relevance of pharmacokinetic interactions. Clin Microbiol Infect, 2006; 12:97-106.

Farooq U, Khan S, Nawaz S, Ranjha NM, Haider MS, Khan MM, Dar E, Nawaz A. Enhanced gastric retention and drug release via development of novel floating microspheres based on Eudragit E100 and polycaprolactone: synthesis and in vitro evaluation. Des Monomers Polym, 2017; 20(1):419-33.

Fartyal S, Jha SK, Karchuli MS, Gupta R, Vajpayee A. Formulation and evaluation of floating microspheres of boswellic acid. Int J Pharm Tech Res, 2011; 3:76-81.

Gajra B, Dalwadi C, Patel R. Formulation and optimization of itraconazole polymeric lipid hybrid nanoparticles (Lipomer) using box behnken design. DARU J Pharm Sci, 2015; 23(1):3.

Garud N, Garud A. Preparation and in-vitro evaluation of metformin microspheres using non-aqueous solvent evaporation technique. Trop J Pharm Res, 2012; 11(4):577-83.

Gaur PK, Mishra S, Bajpai M. Formulation and evaluation of controlled-release of telmisartan microspheres: in vitro/in vivo study. J Food and Drug Anal, 2014; 22(4):542-8.

Gupta R, Prajapati SK, Pattnaik S, Bhardwaj P. Formulation and evaluation of novel stomach specific floating microspheres bearing famotidine for treatment of gastric ulcer and their radiographic study. Asian Pac J Trop Biomed, 2014; 4(9):729-35.

Hao J, Wang F, Wang X, Zhang D, Bi Y, Gao Y, Zhao X, Zhang Q. Development and optimization of baicalin-loaded solid lipid nanoparticles prepared by coacervation method using central composite design. Eur J Pharm Sci, 2012; 47(2):497-505.

Jagtap YM, Bhujbal RK, Ranade AN, Ranpise NS. Effect of various polymers concentrations on physicochemical properties of floating microspheres. Indian J Pharm Sci, 2012; 74(6):512.

Kawashima Y, Niwa T, Takeuchi H, Hino T, Itoh Y. Hollow microspheres for use as a floating controlled drug delivery system in the stomach. J Pharm Sci, 1992; 81(2):135-40.

Kawashima Y, Niwa T, Takeuchi H, Hino T, Ito Y. Preparation of multiple unit hollow microspheres (microballoons) with acrylic resin containing tranilast and their drug release characteristics (in vitro) and floating behavior (in vivo). J Control Release, 1991; 16(3):279-89.

Liu R, Wang S, Fang S, Wang J, Chen J, Huang X, He X, Liu C. Liquid crystalline nanoparticles as an ophthalmic delivery system for tetrandrine: development, characterization, and in vitro and in vivo evaluation. Nanoscale Res Lett, 2016; 11(1):254.

Maghraby GM, Alomrani AH. Synergistic enhancement of itraconazole dissolution by ternary system formation with pluronic F68 and hydroxypropylmethylcellulose. Sci Pharm, 2009; 77(2):401-18.
Mishra B, Reddy KH, Manikanta A, Anand A, Raju MSK. Formulation and optimization of clarithromycin loaded with pullulan acetate microsphere for sustained release by response surface methodology. Int J Drug Dev Res, 2016; 8:011-5.

Nila MV, Sudhir MR, Cinu TA, Aleykutty NA, Jose S. Floating microspheres of carvedilol as gastro retentive drug delivery system: 32 full factorial design and in vitro evaluation. Drug Deliv, 2014; 21(2):110-7.

Nour SA, Shawky Abdelmalak N, Naguib MJ. Bumadizone calcium dihydrate microspheres compressed tablets for colon targeting: formulation, optimization and in vivo evaluation in rabbits. Drug Deliv, 2015; 22(3):286-97.

Pachuau L, Mazumder B. A study on the effects of different surfactants on Ethylcellulose microspheres. Int J Pharm Tech Res, 2009; 1(4):966-71.

Patel A, Ray S, Thakur RS. Invitro evaluation and optimization of controlled release floating drug delivery system of metformin hydrochloride. DARU J Pharm Sci, 2006; 14(2):57-64.

Pandav S, Naik J. Preparation and in vitro evaluation of ethylcellulose and polymethacrylate resins loaded microparticles containing hydrophilic drug. J Pharm, 2014; 2014.

Raut NS, Somvanshi S, Jumde AB, Khandelwal HM, Umekar MJ, Kotagale NR. Ethyl cellulose and hydroxypropyl methyl cellulose buoyant microspheres of metoprolol succinate: Influence of $\mathrm{pH}$ modifiers. Int J Pharma Investig, 2013; 3:163-70.

Raghuvanshi S, Pathak K. Bioadhesive floating microsponges of cinnarizine as novel gastroretentive delivery: Capmul GMO bioadhesive coating versus acconon MC 8-2 EP/NF with intrinsic bioadhesive property. Int J Pharma Investig, 2016; 6:181-93.

Rohilla S, Bhatt DC, Gupta A. Therapeutic potential of phytomedicines and novel polymeric strategies for significant management of candidiasis. Curr Pharm Design, 2018; 24(16):1748-65.

Rowe RC, Sheskey PJ, Owen SC. Handbook of pharmaceutical excipients. Pharmaceutical Press, London, UK, 2006.

Sato Y, Kawashima Y, Takeuchi H, Yamamoto H. Physicochemical properties to determine the buoyancy of hollow microspheres (microballoons) prepared by the emulsion solvent diffusion method. Eur J Pharm Biopharm, 2003; 55(3):297-304.

Sato Y, Kawashima Y, Takeuchi H, Yamamoto H. In vitro evaluation of floating and drug releasing behaviors of hollow microspheres (microballoons) prepared by the emulsion solvent diffusion method. Eur J Pharm Biopharm, 2004; 57(2):235-43.

Shah M, Pathak K. Development and statistical optimization of solid lipid nanoparticles of simvastatin by using 23 full-factorial design. AAPS Pharmscitech, 2010; 11(2):489-96.

Sinko PJ. Micrometrics. In: Martin A (ed). Martin's physical pharmacy and pharmaceutical science. 5th edition, Lippincott Williams and Wilkins, Baltimore, MD, 2006.

Tadros MI. Controlled-release effervescent floating matrix tablets of ciprofloxacin hydrochloride: Development, optimization and in vitro-in vivo evaluation in healthy human volunteers. Eur J Pharm Biopharm, 2010; 74(2):332-9.

Umamaheswari RB, Jain S, Tripathi PK, Agrawal GP, Jain NK. Floating-bioadhesive microspheres containing acetohydroxamic acid for clearance of Helicobacter pylori. Drug Deliv, 2002; 9(4):223-31.

Wang AJ, Lu YP, Sun RX. Recent progress on the fabrication of hollow microspheres. Mater Sci Eng A, 2007; 460:1-6.

How to cite this article:

Rohilla S, Bhatt DC, Ahalwat S. Fabrication of potential gastroretentive microspheres of itraconazole for stomachspecific delivery: Statistical optimization and in vitro evaluation. J Appl Pharm Sci, 2020; 10(03):119-127. 